\title{
Business models for the bioentrepreneur
}

\section{The IT industry could provide useful models for biotechnology ventures.}

\section{Jean-François Formela}

There has been a long debate in the biotechnology industry as to whether to go for product(s) (i.e., vertical applications) or for technology and tools. Clearly, the companies that have delivered the highest rates of return lately have been technology companies. In 1995, only five such "discovery" companies were in the top 100 biotechnology companies and none in the top tier (more than $\$ 1$ billion market value). In 1997, 14 were in the top 100 and two in the top tier'. However, as there is often no simple binary answer to business model discussions, some of these companies may have to become more vertical to support further and continued growth. Interestingly, the increasing analogies between the information technology (IT) and biotechnology industries could provide some directions for emerging business models in the latter.

\section{Making IT pay}

Today, the IT market structure is shaped by successive technology waves that typically emerge as new platforms leading to the emergence of platform specific tools and then the development of vertical applications. Large IT buyers prefer to buy from platform vendors because they integrate several technologies into a solution, thus eliminating the need to go to multiple vendors. Microsoft (Redmond, WA) and others have relied not only on their original technology but also on tools and components bought in the open market as they became dominant. Many large IT companies have followed the same model.

This market structure has provided liquidity in the IT mergers and acquisitions (M\&A) market. Last year, M\&A outnumbered IPOs by 8 to 1 for private companies in the IT sector. The point is that for IT investors and management teams, M\&A have become a common and desirable exit route.

The biotechnology industry-and possibly its most significant technology wave, genomics- has now moved beyond the plat-

Jean-François Formela is a general partner at Atlas Venture, 222 Berkeley Street, Boston MA 02116 (jfformela@atlasventure.com).

\section{New companies are focusing not on specific vertical applications, but providing technology to their customers.}

form phase. Some of the early genomics companies have become platform companies through a combination of timing and critical mass; they are now consolidating their position through system integration. As yet, there is no standard, because the technology is still immature; some in big pharma are looking forward to the time when a standard operating system emerges and fewer vendors provide comprehensive solutions.

Most biotechnology tool/platform companies have now moved away from biology. They are providing data generation tools to their customers, not vertical knowledge of a specific field of biology. Some companies have also started to add multiple components to become system integrators. Good examples of such transactions are Incyte (Palo Alto, CA)-Synteni (Fremont, CA), Millennium-Chemgenics (Cambridge, MA), and Arris (S. San Francisco, CA)-Sequana (La Jolla, CA).

At the same time, instrumentation companies that traditionally were way downstream of biology are now integrating upstream and looking for "software." Hewlett Packard (Palo Alto, CA), for example, is interested in getting access to new chemistry and/or biological information sets that could become the software component of their mass spectroscopy instrumentation and increase its value. The Pharmacia (Uppsala, Sweden)-Amersham (Amersham, UK) and Perkin Elmer (Foster City, CA)-PerSeptive (Cambridge, MA) transactions are consistent with this trend. Many of these transactions are driven by the need to develop standards.

\section{Four business models}

A recent Forbes article referred to "a new generation of biotechnology companies" that are focusing not on specific vertical applications, but providing technology to their customers.
Their business models can be organized in different groups.

First a purely horizontal, nonexclusive model is best exemplified by Incyte. As a dominant content provider, it has been able to produce recurring revenues. The challenge is to sustain uniqueness in order to support pricing.

Affymetrix (Santa Clara, CA) and Perkin Elmer are good examples of the pure tool/component model. Their aim is to create and protect a standard. The ABI 377 has become the standard in its space and Affymetrix could be pursuing the "Intel inside" approach. The challenge is to develop and defend a standard in a complex, fastmoving and immature environment

System integration is yet another approach, best represented by Millennium, that could be characterized as a FIDCO or fully integrated discovery company. New tools are integrated into a platform that can be licensed to customers for use on specific applications in their vertical space(s) (i.e., disease $\mathrm{x}$ within biology $\mathrm{z}$ ). The challenge here is obviously to stay competitive across multiple technologies.

Last but not least is the fully integrated pharmaceutical company or FIPCO. HGS is betting that its platform will change the odds and enable a more efficient discovery process.

\section{Conclusions}

We may see the emergence of a buyer-driven market for biotechnology M\&A transactions. Indeed, the number of such transactions has been steadily growing to 66 last year. It is still far from the IT ratio, but the emergence of true platform companies in biotechnology with large market capitalization should enable new business models to emerge. In the IT world, industrial end users did not build their own IT proprietary tools by internal development or acquisitions, rather relying on technology providers. It will be interesting to observe whether these changes in market structure will have an impact on private biotechnology companies' exit strategy.

1. Hambrecht \& Quist. 1997. Private Report: Top 100 biotechnology company index. 